\title{
Calculating the Speed of Vehicles Using Wireless Sensor Networks
}

\author{
Omar Alfandi*, Arne Bochem, Mehdi Akbari Gurabi, \\ Alberto Rivera Díaz, Md.Istiak Mehedi, Dieter Hogrefe \\ Institute of Computer Science, University of Göttingen, Germany \\ *College of Technological Innovations, Zayed Universtiy, United Arab Emirates \\ Email: \{alfandi,bochem,hogrefe\}@ cs.uni-goettingen.de, \\ \{mehdi.akbarigurabi, alberto.riveradiaz, mdistiak.mehedi\}@stud.uni-goettingen.de
}

\begin{abstract}
Speed measurement is an important issue for some types of Wireless Sensor Networks (WSN), especially for Vehicular Ad-hoc Networks (VANETs). However, calculating this value is error-prone and costly. This report intends to demonstrate the calculation of speed of an object without the use of any additional devices or sensor boards, only using Received Signal Strength Indication (RSSI) for localization of the vehicles and time calculation using synchronization. We implemented these methods in actual IRIS motes, and tested them. The results show that, while not perfectly accurate, our method proved to be reliable and close to the real speed. In addition, the results do not have any linear correlation in divergence of real speed and calculated speed, which means the system avoids systematic errors.
\end{abstract}

\section{INTRODUCTION}

$\mathbf{N}$ OWADAYS, Wireless Sensor Networks form a big part of our networks and their utilization has seen a rapid increase. This growth is effected due to newly developed wireless multimedia services and attributions such as data, voice and video [1]. In this way, WSN have started to play a significant role in our everyday life. They have a wide range of applications, from monitoring the environment and the human body to industrial and military applications which are slowly becoming ubiquitous [2].

Mobility is a major issue for several WSN applications. An example of a useful application is in the branch of VANETs, a subclass of Mobile Ad-hoc Networks (MANETs), which relies on vehicles to provide functionality. Their approach is of great importance to Intelligent Transportation Systems (ITS). The main differences between normal MANETs and VANETs are the mobility constraints, high mobility, and the driver's behaviour all of which are found in the latter [3]. VANETs provide a variety of applications that ranging from safety solutions, collision detection and crash avoidance to Internet access and multimedia [4]. These applications are expected to be deployed on a larger scale in the future due to their usefulness, for example in providing safety to drivers or enhancing inter-vehicle communication [1].

VANETs make use of several traffic flow parameters to perform the monitoring, such as speed of the vehicle. Several methods have been implemented to calculate this parameter, for example: Global Positioning System (GPS)localization[5], the Laser Doppler Techniques[6], or Received-Signal-Strength(RSS)[5]. Most of these methods need additional devices for their calculation such as directional antenna [7] or GPS module that can increase the cost of deployment.

In this report, a simple method using RSSI according to characteristics and attributes of VANETs mobility model was implemented. We are modelling a street by using some IRIS motes as fixed nodes for gathering data as the infrastructure of the network, and send information of cars to our base station. The base station calculates the velocity of cars by means of this data.

The paper is organized in seven sections: Section 2 discusses related works. Section 3 explains the methodology. In section 4, various advantages and disadvantages of the chosen approach are discussed. Section 5 explains further challenges. Results of experimental measurements are provided in section 6. In section 7 conclusions are drawn.

\section{RELATED WORKS}

There exist several ways to measure the speed of a moving object. This is of particular interest to law-enforcement for speed limits of vehicles on the roads. The most popular and widespread method is through the use of radio signals.

In this section previous approaches and works on this topic are highlighted.

\section{A. Doppler Effect}

Doppler radar speed measuring unit: Radar guns or speed guns are commonly used in the enforcement of speed limits on highways. They employ the doppler effect to measure the speed of moving objects by detecting a change in frequency of the returned radar signal. From the difference in frequency, the speed of the object can be calculated.

There are two types of speed guns[6]: Stationary and moving radar. For the stationary radar, a signal with a frequency equal to this difference is created by mixing the received radio signal with a little of the transmitted signal. These two radio signals are mixed to create a "beat" signal (called a heterodyne) and an electrical circuit that measures this frequency using a digital counter and displays the number on a digital display as the object's speed. In moving radars, a gun receives reflected signals from both the target vehicle and stationary background objects such as the road surface, nearby road signs, guard rails and street light poles. 
Although very useful, this method has several drawbacks. In order to function correctly, radio waves must leave the gun in a narrow beam that do not spread out much to avoid receiving a false return from nearby objects or vehicles. Another disadvantage, is that user training and certification are required so that a radar operator can use the equipment effectively. They also do not work very well in traffic and significant vehicle separation is needed to ensure an accurate measurement.

\section{B. GPS}

GPS tracking units: The Global Positioning System is another technology that can be utilized to obtain the speed of a vehicle. It provides time and location information, regardless of the weather, or the location on Earth. A GPS tracking unit is a device that uses the Global Positioning System to determine the precise location of a vehicle, person, or other objects; to which it is attached and to record the position of the asset at regular intervals. The recorded location data can be stored within the tracking unit, or it may be transmitted to a central location data base, or internet-connected computer, using a cellular (GPRS or SMS), radio, or satellite modem embedded in the unit.

One type of GPS tracking unit is the data pusher; that is used for asset tracking, personal tracking and Vehicle tracking systems. Also known as a GPS beacon, this kind of device sends the position of the device as well as other information to a determined server to be stored and analysed. By knowing the exact location of a moving object, its speed can be easily calculated, thus making GPS a tool for obtaining the said value.

\section{Other methods}

LiDAR speed gun: LiDAR is a remote sensing technology that measures distance by illuminating a target with a laser and analysing the reflected light. The term is a portmanteau of "light" and "radar." The police uses LiDAR[6] speed guns for speed limit enforcement, which in turn use LiDAR technology to detect the speed of a vehicle. Unlike radar speed guns, which rely on doppler shifts to measure the speed of a vehicle, these devices allow a police officer to measure the speed of an individual vehicle within a stream of traffic.

RSSI: RSS is defined as the voltage measured by a receiver's received signal strength indicator (RSSI) circuit. Often, RSS is equivalently reported as measured power, i.e., the squared magnitude of the signal strength. We can consider the RSS of acoustic, RF, or other signals. Wireless sensors communicate with neighbouring sensors, so the RSS of RF signals can be measured by each receiver during normal data communication without presenting additional bandwidth or energy requirements. RSS measurements are relatively inexpensive and simple to implement in hardware. Some approaches used for localization are Time of Arrival (ToA) [5], Angle of Arrival (AoA) [7] and Triangulation Method [8].

The Infra-Red Traffic Logger: The Infra-Red Traffic Logger, more commonly known simply by the acronym TIRTL, is a multi-purpose traffic sensor that can be used as a traffic counter, speed sensor, red light camera sensor, heavy vehicle tracker, over-height vehicle sensor, rail crossing sensor and network management system.
The system consists of a receiver unit and transmitter unit placed on opposite sides of the road perpendicular to the direction of travel. The transmitter sends two cones of infrared light across the roadway, and the receiver records vehicles as they break and remake these cones [9].

\section{Chosen approach}

This project aims at demonstrating how to calculate the speed of an object, particularly a vehicle, which moves from one point to another by using only traditional wireless sensor motes without the aid of any specialized sensor boards. In order to obtain said speed, two measures are required: distance and time elapsed. For our project, the distance is a set value, which is given by the distance between two fixed nodes. The time elapsed however, is the main concern. It is calculated by using the RSSI approach to obtain the approximate point in time and space in which the vehicle passes through a fixed point. The higher the received signal, the closer the vehicle is to the fixed point. This allows calculating the difference in times between the nodes, and thus, obtain also the total time elapsed while the vehicle stayed between these fixed points. Implementing other procedures such as GPS to obtain the location of the moving nodes results in high costs and is not energy efficient. Therefore it is intended to demonstrate that a simpler and cheaper approach can be applied with approximately equal effectiveness, rather than a costly and complex one such as GPS or radar based systems.

\section{Methodology}

In this section, the methodology and experimental setup will be described in details.

\section{A. Lab setup}

Sending nodes: These are the main target of the project. A wireless mote is attached to the vehicles or moving objects. It will be constantly sending dummy packets, which also contain the nodes ID to be processed by the fixed nodes. The

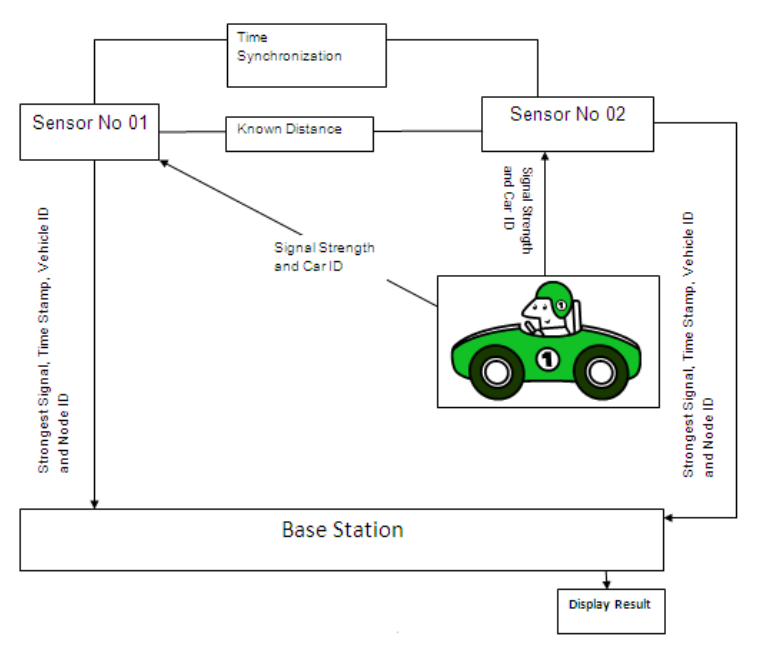

Fig. 1: Overview diagram. 


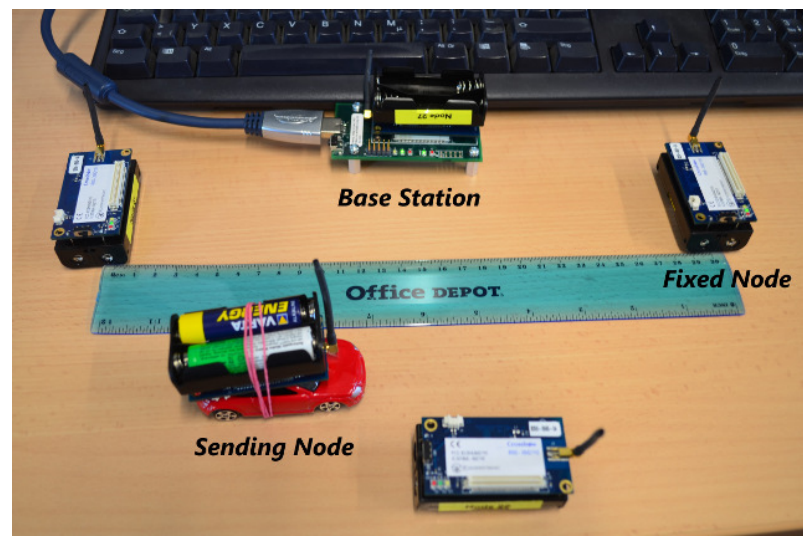

Fig. 2: Lab setup.

ID distinguishes all the different moving nodes. The speed measurement obtained at the end of the process is calculated based on the linear speed of this node assuming it has a constant speed and moves in a linear path. It then sends the packets at a high speed in order to achieve a higher accuracy.

Fixed node: Its function is to receive data, i.e. sense the moving motes and receive their ID and send it to the Base Station. In these motes, the RSSI for the packets sent by vehicular nodes is obtained, as well as the time-stamp of the moment in which the packets are received from the moving mote. Only the strongest signals are chosen to be sent to the Base Station together with the ID and time of receipt. These motes are scattered across the lane and have a fixed distance between them. This allows for the calculation of the speed of the vehicle in a portion of the road, between two fixed nodes.

Base station: The base station gathers the data from the fixed points and makes decisions based on the first packet having the signal strength of the moving mote. Using this information, a graphical interface is deployed to calculate the speed of the vehicle by filtering the data received and finally displaying the results.

\section{B. Process}

1. Sending packets: The mobile sending motes are the representation of the vehicles in a highway. These nodes are flashed with a program that executes the following functions: First, when the node is booted, the radio capability is enabled. This will allow communication between the node and the fixed points. Afterwards, packets are assembled by obtaining the nodes ID which will be used to identify the vehicle or object among all the different nodes. These packets are then continuously broadcast.

2. Receiving packets: Fixed motes are scattered across the lane or path which the mobile nodes pass by. They represent the second stage of the process and are flashed with a program called RssiBase that performs the following actions:

When a fixed node receives a packet from a sending node, the fixed node starts preparing a new packet with the information obtained from the sending node, that is the nodes ID, and two new values: the time-stamp and the Received Signal Strength Indication (RSSI) from said mote, which are calculated at the time of reciept. This time is synchronized by applying the Flooding Time Synchronization Protocol(FTSP).

The RSSI value of the sending nodes is used to determine when a mobile node gets to the closest point to the fixed node. When this happens, a time-stamp will allow for the calculation of the total time elapsed between fixed nodes, which is one of the values required in the formula of speed. Therefore, only the values with maximum RSSI received must be used. The fixed node calculates this from a set number of packets, which are received and compared to the previous RSSI value to determine if it is higher or not. If the new value received is higher, then it is stored along with its node ID. After a maximum amount of packets received is reached, the values are encapsulated into a packet and broadcast in order to be received by the base station.

3. Gathering data: The base station receives the packets which contain the highest RSSI received at each fixed node, along with the associated ID and timestamp. The base station mote additionally participates in time synchronization by means of the FTSP protocol.

4. Calculating and displaying the results: After gathering the information needed from all the fixed notes, the results are displayed. This is achieved by a Java application running on a PC, to which the base station is attached. It obtains the data collected from the base station and displays it in a graphical user interface. The actions executed by this program are described in the following steps:

A packet is received at the base station. First, any packets that are useless to the application are filtered out. This avoids cases like getting a second reading from the same car and same fixed node, to preserve the order of events, which could confuse calculation.

There is also another filter that checks if all the packets from the participant fixed nodes have been sent and received by the base station. Calculations can only be done when the condition is met.

After passing both filters, the elapsed time is calculated. This value is obtained by subtracting the time-stamp of the last fixed node's packet from the previous fixed node's packet, and so forth. This will result in the total time between the first and last fixed node.

The elapsed time, along with the vehicle's ID are then passed down to another function within the program, which finally calculates the vehicle's speed.

The common formula to calculate velocity is applied inside that function:

$$
v=\frac{d}{t}
$$

Here, "d" is the distance travelled, and "t" elapsed time. For the distance value, since the locations of fixed nodes are known, the travelled distance is simply found as shown in figure 4. A fixed distance is set before running and compiling the Java program, and is dependent on the distance between fixed nodes.

After obtaining the speed value, the speed and node ID are displayed in the GUI. (Results are given in $\mathrm{m} / \mathrm{s}$.) This process 


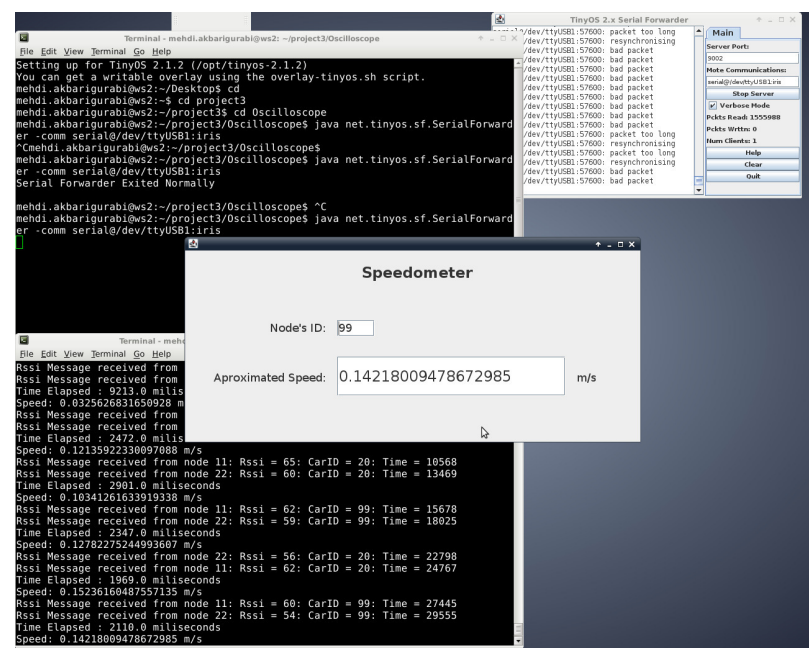

Fig. 3: Results screen.

will repeat indefinitely every time a vehicle passes by the fixed nodes from start to end.

\section{EVAluATION OF THE APPROACH AND CHALLENGES}

In the following, advantages and disadvantages of the described approach are explained.

\section{A. Advantages}

Cheaper and easier deployment compared to other approaches that require expensive or complicated tools. By using only the RSSI of the moving nodes, the time elapsed from a fixed point to another can be calculated without the need of special sensor boards or complex schemes to determine the exact moment when the node passes by a point.

By avoiding the use of additional devices, battery consumption is reduced, as opposed to cases where more powerdemanding sensors are required. This also translates to lower costs spent in energy and maintenance.

According to our measurements, this approach has reasonably good accuracy.

\section{B. Disadvantages}

The approach also has some disadvantages and drawbacks, as explained in the following:

The vehicle or moving object requires a wireless sensor mote. This is the biggest drawback of the project since it will only calculate the speed of an object that has a wireless sensor mote attached to it. If it were to be implemented in a real scenario, vehicle manufacturers would have to be required to implement a similar device which can transmit similar packets. Alternatively, wireless devices of this type could be distributed to vehicle owners. Another possibility that could be investigated would be the use of ubiquitous smart phones as a packet source.

In free space, signal power decays proportional to $d^{2}$, where $\mathrm{d}$ is the distance between the transmitter and receiver.
In real-world channels, multipath signals and shadowing are two major sources of environmental influence on the measured RSS.

Multiple signals with different amplitudes and phases arrive at the receiver, and these signals add constructively or destructively as a function of the frequency, causing frequencyselective fading [5].

These factors may lead to lower accuracy in real world scenarios.

\section{Challenges}

As of now, this project is very limited due to the magnitude of implementing a real life system where actual cars and fixed points are used. It can be considered a proof of concept to be built upon in future works. As explained in the previous section, several physical constraints appear.

One of the problems with this approach is that the speed of cars could surpass that which our current motes can send at. Packets can get lost easily, not arrive at the correct time or not arrive at all at the fixed nodes, which would lead to incorrect calculations. A solution to this would be deploying more powerful devices with a better transmitting power range.

Another challenge to be tackled is the scalability of the project. For simplicity reasons, we have only implemented a two-component system where one or several moving nodes pass by only two fixed nodes. Real world implementation would require several fixed nodes to have a practical use. This is because the distance between motes has to be within their transmitting range, which limits the distance for a given measurement. Therefore several fixed motes should be employed to be within the range of the network and measure a much greater distance in a highway or path for the purpose of increasing accuracy.

\section{RESUlTS AND DISCUSSION}

In the following, the testbed is described and experimental measurements are given.

\section{A. Test setup}

To prove the effectiveness and accuracy of our project, several tests were performed. A ruler was used to set the exact distance between the fixed nodes. The ground truth is measured using a stop watch. Two IRIS nodes are used as fixed nodes, one IRIS node is attached to a toy car and one acts as a base station.

\section{B. Steps}

The system was tested by flashing all the motes with their respective programs, so they could perform their respective duties. The sending mote was attached to a toy car, and proceeded to place both of the fixed nodes at a fixed distance. We tested with four different distances. First, a distance of $30 \mathrm{~cm}$, then $60 \mathrm{~cm}, 100 \mathrm{~cm}$, and finally $200 \mathrm{~cm}$ were tested. We tried the system with different random paces: slow (around $0.2 \mathrm{~m} / \mathrm{s}$ ), medium (around $0.5 \mathrm{~m} / \mathrm{s}$ ) and fast (around $1 \mathrm{~m} / \mathrm{s}$ ). We test the system 10 times for each distance and speed. The measurement of time was done with a stopwatch, starting and 


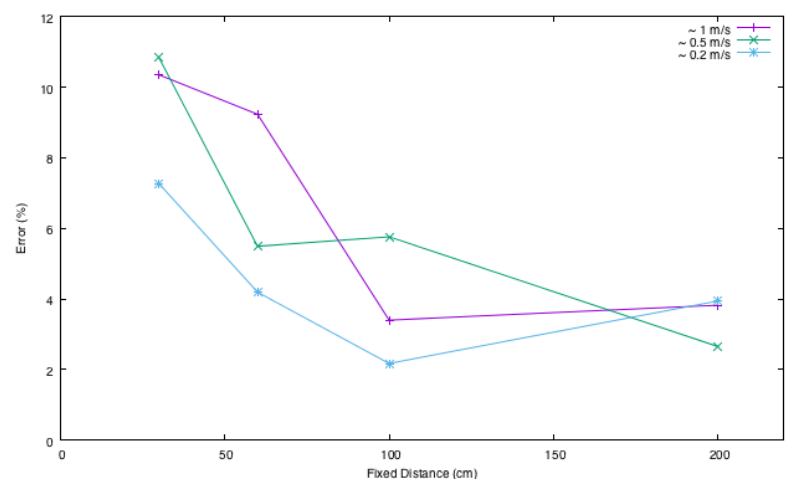

Fig. 4: Accuracy of speed calculations, Error percentage $=100 *\left|\frac{v_{\text {real }}-v_{\text {calc }}}{v_{\text {real }}}\right|$

ending the timer whenever the sending mote passed next to one fixed node. This allowed us to obtain the total time elapsed. At the end of each test, the manual results obtained from our calculations (The real, accurate speed) with the results displayed on screen given as output from our program.

\section{Results}

Our results are shown in figure 4. From these results, it is observed that the error percentage between the manually measured values and the values obtained with our application is very small. The most accurate result obtained had an error of $0.94 \%$, whereas the least accurate result had an error of $14.86 \%$. This is out of 120 tests performed which is averaged from 10 tests per each pair of distance and speed.

Another observation is that the set of tests with the shortest distance between fixed nodes $(0.30 \mathrm{~m})$ has the least accurate results, whereas the set of tests with the longer distances ( $1 \mathrm{~m}$ or $2 \mathrm{~m}$ ) has the most accuracy. This can indicate that the closer the fixed nodes are, the less accurate calculations are obtained, possibly due to the packet's arrival times at the Base Station. Shorter paths mean that packets may arrive at close or same time. Additionally, over longer distances, small errors in measurement time will have less influence than equal errors over short distances, because the total amount of time is higher and, overall, outweighs it.

\section{CONCLUSION}

The approach used in this project to calculate the speed of a vehicle using wireless sensor networks proved to be effective and reasonably reliable as shown in the results of the testing. Calculating the time elapsed without the aid of any extra devices or sensor boards is a big advantage, compared to the higher costs of deploying a network of several more powerconsuming and expensive devices, as the RSSI of a wireless mote can be obtained without specialized sensor boards. The results show that our method proved to be reliable and close to the real velocity, and no linear correlation in difference of real and measured velocity shows that the speed measurement is free of systematic errors.

In the future, the approach can be extended in various ways, such as including more fixed nodes, using commonly available devices such as smartphones for the mobile node role and scaling everything up to a real world scenarios outside of a controlled testbed.

\section{REFERENCES}

[1] E. Spaho, L. Barolli, G. Mino, F. Xhafa, and V. Kolici, "Vanet simulators: A survey on mobility and routing protocols." Broadband and Wireless Computing, Communication and Applications (BWCCA), 2011 International Conference on (pp. 1-10). IEEE., 2011.

[2] I. F. Akyildiz, W. Su, Y. Sankarasubramaniam, and E. Cayirci, "A survey on sensor networks." Communications magazine, IEEE, 40(8), 102-114., 2002.

[3] S. Yousefi, M. S. Mousavi, and M. Fathy, "Vehicular ad hoc networks (vanets): challenges and perspectives." ITS Telecommunications Proceedings, 2006 6th International Conference on (pp. 761-766). IEEE., 2006.

[4] G. M. Abdalla, M. A. Abu-Rgheff, and S. M. Senouci, "Current trends in vehicular ad hoc networks." Ubiquitous Computing and Communication Journal, 1-9., 2007.

[5] N. Patwari, J. N. Ash, S. Kyperountas, A. O. Hero, R. L. Moses, and N. S. Correal, "Locating the nodes: cooperative localization in wireless sensor networks," Signal Processing Magazine, IEEE, 22(4), 54-69., 2005.

[6] M. Adnan, N. Zainuddin, N. Sulaiman, and T. Besar, "Vehicle speed measurement technique using various speed detection instrumentation," in Business Engineering and Industrial Applications Colloquium (BEIAC), 2013 IEEE, April 2013, pp. 668-672.

[7] J. R. Jiang, C. M. Lin, F. Y. Lin, and S. T. Huang, "Alrd: Aoa localization with rssi differences of directional antennas for wireless sensor networks," Information Society (i-Society), 2012 International Conference on(pp. 304-309). IEEE., 2012.

[8] Z. Li, W. Trappe, Y. Zhang, and B. Nath, "Robust statistical methods for securing wireless localization in sensor networks," Information Processing in Sensor Networks, 2005. IPSN 2005. Fourth International Symposium on, 2005.

[9] TIRTL, http://www.ceos.com.au/index.php/products/tirtl, 2014, [Accessed October 5, 2014]. 